Übersichten

Monatsschr Kinderheilkd 2023 $\cdot 171: 302-313$ https://doi.org/10.1007/s00112-020-01027-2 Online publiziert: 28. September 2020

(c) Der/die Autor(en) 2020

\section{Redaktion}

B. Koletzko, München

T. Lücke, Bochum

E. Mayatepek, Düsseldorf

N. Wagner, Aachen

S. Wirth, Wuppertal

F. Zepp, Mainz

\section{N. Bruns · C. Dohna-Schwake ${ }^{1,2}$}

'Zentrum für Kinder- und Jugendmedizin, Klinik für Kinderheilkunde I, Universitätsmedizin Essen, Essen, Deutschland

${ }^{2}$ Westdeutsches Zentrum für Infektiogie, Universitätsmedizin Essen, Essen, Deutschland

\title{
Antibiotische Therapie bei kritisch kranken Kindern - Ist weniger mehr?
}

\section{Ein narratives Review aktueller Studien und Bezugnahme auf die aktuelle Leitlinie der Surviving Sepsis Campaign}

\section{Hinleitung}

Durch den Einsatz von Antibiotika können schwere Infektionen geheilt und damit Leben gerettet werden - dennoch sollten sie nur verabreicht werden, wenn von ihrem Einsatz tatsächlich ein Nutzen zu erwarten ist. Die Entscheidung für oder gegen eine antibiotische Therapie bzw. zu ihrer Beendigung ist im klinischen Alltag allgegenwärtig. Insbesondere bei kritisch kranken Kindern wird sie jedoch nicht ausschließlich nach rationalen Gesichtspunkten getroffen.

\section{Hintergrund}

Die Weiterentwicklung der Medizin hat in den Industrieländern $\mathrm{zu}$ einer $\mathrm{Zu}$ nahme von chronisch kranken Kindern und Jugendlichen geführt, die anfällig für Infektionen sind. Beispielhaft seien die Absenkung der Grenze der Überlebensfähigkeit bei extremer Frühgeburtlichkeit, die Durchführung von Organund Knochenmarktransplantationen sowie die deutlich verbesserte Prognose von Patienten mit zystischer Fibrose genannt. Dies führt zu einer Zunahme infektiologischer Fälle auf pädiatrischen Intensivstationen: Eine retrospektive Studie aller Kinderintensivstationen Australiens und Neuseelands zeigte eine zunehmende Prävalenz invasiver Infektionen bei
Aufnahme auf die Intensivstation, bei sinkender Mortalität [36].

Die Entscheidung für eine antibiotische Therapie wird auf der Grundlage einer lokalen oder systemischen Inflammation (Fieber, Tachykardie, Tachypnoe) in Kombination mit laborchemischen Biomarkern (Leukozytenzahlen, C-reaktivem Protein [CRP] oder Prokalzitonin [PCT]) getroffen. Bei immunsupprimierten oder chronisch kranken Kindern und Jugendlichen können die klinischen Symptome subtiler und die laborchemische Entzündungsreaktion weniger ausgeprägt sein oder fehlen. Zudem können mit einer bakteriellen Infektion vereinbare Symptome und laborchemische Veränderungen anderweitig verursacht sein: virale Infektionen, autoimmunologische Prozesse, Grunderkrankungen wie Leukämie oder andere Erkrankungen wie Subileus oder Pankreatitis führen zu systemischer Inflammation und können eine bakterielle Infektion imitieren. Bei diesen Patienten wird die Indikation für eine antibiotische Therapie großzügig gestellt, da die Folgen einer unbehandelten schweren bakteriellen Infektion im Sinne einer schweren Sepsis oder eines septischen Schocks gefürchtet sind.

In pädiatrischen Risikopopulationen ist die Rate von Antibiotikatherapien hoch, wie verschiedene Punktprävalenzerhebungen zeigen: Eine europäische Studie (ARPEC: Antibiotic Resistance and Prescribing in European Children) stellte eine konstant hohe Zahl von antibiotischen Therapien, inklusive Breitspektrumantibiotika, in der stationären Kinderheilkunde auf 4 verschiedenen Kontinenten fest: $37 \%$ aller stationär aufgenommen Patienten erhielten eine antimikrobielle Therapie [48]. In spezialisierten Bereichen wie der pädiatrischen Intensivmedizin liegt die Prävalenz antibiotischer Therapien bei bis $\mathrm{zu} 79 \%$, davon sind bis $\mathrm{zu} 62 \%$ inadäquat [4]. Auch bezüglich der perioperativen Prophylaxe entspricht die Verabreichung von Antibiotika häufig nicht den Leitlinien [16]. Die Initiierung einer antibiotischen Therapie ist dabei nicht immer nur an klinischen Parametern orientiert, sondern durch die Erwartungshaltung an den Behandler bzw. die Angst oder Sorge des Behandlers mitbeeinflusst. Exemplarisch sei hier ein Zitat aus einer qualitativen Untersuchung zu Einflussfaktoren antibiotischer Therapie bei Ärzten anhand qualitativer Fragebogen erwähnt: „When it is 3:00 in the morning, depending on how busy you are, the easiest solution is to throw vancomycin and piperacillin-tazobactam at every patient because you do not have time to read the confusing guidelines that tell you 16 different things you would potentially do" (Resident interview; [24]). 
Hier steht eine Anzeige.

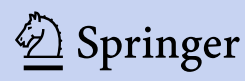


Tab. 1 Definition wichtiger infektiologischer Begriffe gemäß der Internationalen pädiatrischen Sepsiskonsensuskonferenz von 2005 [14] und der Guideline Surviving Sepsis Campaign von 2020 [50]

\begin{tabular}{|c|c|}
\hline Begriff & Definition \\
\hline \multirow{6}{*}{$\begin{array}{l}\text { "Systemic inflammatory } \\
\text { response syndrome“ (SIRS) }\end{array}$} & 2 oder mehr Kriterien (davon mindestens eins Körpertemperatur oder Leukozytenzahl): \\
\hline & Körperkerntemperatur $>38,5^{\circ} \mathrm{C}$ oder $<36^{\circ} \mathrm{C}$ \\
\hline & $\begin{array}{l}\text { Tachykardie (Herzfrequenz >2 Standardabweichungen über der Altersnorm ohne äußere Stimuli oder Medikamente } \\
\text { ODER eine dauerhafte Erhöhung der Herzfrequenz für mehr als } 30 \mathrm{~min} \text { ohne Erklärung) }\end{array}$ \\
\hline & $\begin{array}{l}\text { Bradykardie (bei Säuglingen <1 Jahr: Herzfrequenz unterhalb der 10. Perzentile ohne Vagusreiz, } \beta \text {-Blocker oder ange- } \\
\text { borene Herzfehler) }\end{array}$ \\
\hline & $\begin{array}{l}\text { Tachypnoe (Atemfrequenz mehr als } 2 \text { Standardabweichungen über der Altersnorm oder akut notwendige maschinelle } \\
\text { Beatmung) }\end{array}$ \\
\hline & $\begin{array}{l}\text { Leukozytenzahl über die Altersnorm erhöht oder erniedrigt (nicht als Folge von Chemotherapie) bzw. mehr als 10\% } \\
\text { Stabkernige }\end{array}$ \\
\hline Infektion & $\begin{array}{l}\text { Vermutete oder bestätigte Infektion ODER eine klinische Symptomkonstellation mit einer hohen Wahrscheinlichkeit } \\
\text { für eine Infektion }\end{array}$ \\
\hline Sepsis $^{\mathrm{a}}$ & SIRS in Kombination mit oder als Folge einer vermuteten oder nachgewiesenen Infektion \\
\hline Schwere Sepsis ${ }^{a}$ & $\begin{array}{l}\text { Sepsis plus kardiovaskuläre Organdysfunktion ODER akute respiratorische Organdysfunktion ODER akutes respiratori- } \\
\text { sches Dysstress-Syndrom ODER mindestens } 2 \text { andere Organdysfunktionen }\end{array}$ \\
\hline Septischer Schock & $\begin{array}{l}\text { Schwere Infektion mit kardiovaskulärer Organdysfunktion (Hypotension trotz Gabe von mindesten } 40 \mathrm{ml} / \mathrm{kg} \text { Infusion, } \\
\text { notwendige Gabe von Vasopressiva, beeinträchtigte Perfusion diagnostiziert anhand von Laktat, base excess, Kapillar- } \\
\text { füllungszeit, Oligurie, rektal-axilläre Temperatur-Differenz) }\end{array}$ \\
\hline $\begin{array}{l}\text { Sepsisassoziierte Organdys- } \\
\text { funktion }\end{array}$ & Schwere Infektion, die zu kardiovaskulärer oder nichtkardiovaskulärer Organdysfunktion führt ${ }^{\mathrm{b}}$ \\
\hline \multicolumn{2}{|c|}{$\begin{array}{l}\text { 'Für Erwachsene wurden } 2016 \text { neue Definitionen und Kriterien für die Begriffe „Sepsis“ und „septischer Schock“ publiziert (Sepsis-3). Der Begriff "schwere } \\
\text { Sepsis" wurde gestrichen und durch eine neue Definition von Sepsis ersetzt [41]. Für die Pädiatrie wurde diese neue Terminologie bisher nicht übernommen } \\
\text { "Für die Guideline der Surviving Sepsis Campaign wurde bewusst keine spezifische Definition für Organdysfunktion gewählt, da es in der Literatur } \\
\text { unterschiedliche Definitionen für die sepsisassoziierte Organdysfunktion gibt. Auch die Definition des septischen Schocks wurde allgemein gehalten [50] }\end{array}$} \\
\hline
\end{tabular}

Die möglichen negativen Folgen einer antibiotischen Therapie sind vielfältig: Nephro- und Ototoxizität von Aminoglykosiden, allergische Reaktionen v.a. bei $\beta$-Lactam-Antibiotika, gastrointestinale Nebenwirkungen und pseudomembranöse Kolitis sowie schwerwiegende Folgen wie das Syndrom des „drug rash with eosinophilia and systemic symptoms" (DRESS) z.B. bei Vancomycin. Die kurz-, mittel- und langfristigen zumeist negativen - Folgen einer Veränderung des Mikrobioms sind noch nicht endgültig geklärt $[23,39,46]$. Eindeutig ist der Zusammenhang zwischen zunehmendem Antibiotikaverbrauch und der Entwicklung von multiresistenten Erregern [18]. Dies gilt sowohl für den individuellen Patienten, dessen Risiko für Infektionen mit multiresistenten Bakterien bei vorheriger Antibiotikaexposition signifikant steigt, als auch für ganze Patientenpopulationen [35]. Bei Erwachsenen mit gramnegativer schwerer Sepsis zeigte sich in einer retrospektiven Studie eine signifikant höhere Sterblichkeit sowie ein signifikant höherer Anteil an resistenten und multiresistenten Erregern, wenn sie in den letzten 90 Tagen vor Beginn der Sepsis Antibiotika erhalten hatten [19]. Infektionen mit multiresistenten Bakterien scheinen das Sterberisiko zu erhöhen, allerdings wird dies nicht konsistent in allen Studien berichtet [11, 29]. Durch die zunehmende Resistenzentwicklung ist eine rationale, zielgerichtete antibiotische Therapie in den Fokus gerückt. Antibiotic-Stewardship(ABS)-Programme sind in zahlreichen Kliniken etabliert worden. Diese Programme senken den Verbrauch von Antibiotika, ohne das Outcome zu verschlechtern $[9,21,25]$.

Die Übertragbarkeit von klinischen Studien im Erwachsenenalter ist limitiert. Dennoch werden einige wegweisende Arbeiten zitiert und wichtige Aspekte der 2020 erschienenen Neufassung der Leitlinien der Surviving Sepsis Campaign berücksichtigt. Wichtige Definitionen infektiologischer Begriffe, die im Rahmen der Internationalen pädiatrischen Sepsiskonsensuskonferenz [14] und der Surviving Sepsis Campaign [51] festgelegt wurden, sind $\bullet$ Tab. $1 \mathrm{zu}$ entnehmen.

\section{Zeitpunkt der antibiotischen Therapie}

\section{Indikation zur sofortigen Therapie}

Unstrittig ist der unverzügliche Beginn einer antibiotischen Therapie bei kreislaufinstabilen Patienten. Grundlage hierfür ist eine retrospektive Studie, die bei erwachsenen Patienten mit septischem Schock eine Verdoppelung der Mortalität bis zur Krankenhausentlassung zeigte, wenn die antibiotische Therapie nicht innerhalb der ersten halben Stunde begonnen wurde. Jede weitere Verzögerung um eine Stunde war im Durchschnitt mit einer Zunahme der Mortalität um $7 \%$ vergesellschaftet [22]. In einer aktuellen Studie erwachsener Patienten mit septischem Schock konnte dieser lineare Zusammenhang nicht nachvollzogen werden, und die Unterschiede zwischen früh und verspätet verabreichter antibiotischer Therapie waren weniger ausgeprägt: Die Krankenhaussterblichkeit betrug $16 \%$ bei Antibiotikagabe innerhalb der ersten Stunde in der Notaufnahme und war gering, aber signifikant erhöht 
bei Verabreichung nach $1-2 \mathrm{~h}$ auf $20 \%$ und auf $22 \%$ nach mehr als $3 \mathrm{~h}$ [20]. In einer retrospektiven Analyse von Kindern mit Bakteriämie in einer britischen Notaufnahme betrug die durchschnittliche Zeit bis zur Verabreichung von Antibiotika 184 min nach erster Vorstellung. Dabei war das klinische Bild bei Healthcareassoziierten Bakteriämien offensichtlich weniger suggestiv für eine invasive Infektion als bei ambulant erworbenen Bakteriämien durch Pneumokokken, Haemophilus influenzae oder Meningokokken: Bei diesen Patienten dauerte es $1 \mathrm{~h}$ länger bis zur Gabe der Antibiotika [17]. Als ursächlich ist hier am ehesten die hohe Virulenz der 3 Letztgenannten zu sehen, die zu einem schwereren Krankheitsbild führt. Mit 140 min etwas kürzer wardieZeit auf einer pädiatrischen Intensivstation vom Erkennen einer schweren Sepsis oder septischem Schock bis zur Gabe eines Antibiotikums. Die kritische Schwelle mit einer signifikant erhöhten Sterblichkeit lag in diesem Studienkollektiv bei $3 \mathrm{~h}$ („odds ratio“ [OR] nach Adjustierung für die Schwere der Erkrankung 4,8 (95\%-Konfidenzintervall (95\%-KI) 1,5-16,2)) [50]. Eine besonders anfällige Patientengruppe für bakterielle Infektionen sind Kinder in Neutropenie nach Chemotherapie. Salstrom et al. konnten durch systematische Schulungen die mittlere Zeit bis zur Antibiotikagabe bei Kindern mit Fieber in der Neutropenie auf unter 60 min senken die Verkürzung der Zeit war mit einer signifikant reduzierten Aufnahmerate auf der Intensivstation assoziiert [32]. Die in dieser Studie identifizierten Hürden für eine frühzeitige Administration waren 1) Wartezeit auf das Blutbild, 2) Transferzeit aus der Aufnahme bis zum Infusionszimmer und 3) Warten auf die Anordnung der Antibiotika durch den Arzt.

Die Wichtigkeit einer schnellen Antibiotikagabe bestätigt sich also auch für pädiatrische Patienten mit febriler Neutropenie und Kinder mit septischem Schock oder Organversagen. Aber kann unter bestimmten Umständen ein abwartendes Verhalten sinnvoll sein?

Monatsschr Kinderheilkd 2023 · 171:302-313 https://doi.org/10.1007/s00112-020-01027-2

(c) Der/die Autor(en) 2020

N. Bruns · C. Dohna-Schwake

Antibiotische Therapie bei kritisch kranken Kindern - Ist weniger mehr? Ein narratives Review aktueller Studien und Bezugnahme auf die aktuelle Leitlinie der Surviving Sepsis Campaign

Zusammenfassung

Die antibiotische Therapie stellt eine wichtige und in vielen Fällen unverzichtbare Maßnahme zum Erreichen einer Restitutio ad integrum bei bakteriellen Infektionen dar. Hierdurch können auch schwere Infektionen bei immungeschwächten Patienten geheilt werden. Wir wissen heute aber auch, dass insbesondere kritisch kranke Kinder häufig inadäquat antibiotisch behandelt werden - mit ebenfalls potenziell schädlichen Nebenwirkungen. In diesem Spannungsfeld aus kritisch krankem Kind, der Angst, etwas zu verpassen, und potenzieller Übertherapie ist es oft nicht einfach, eine rationale Therapieentscheidung zu fällen. Im vorliegenden Review werden aktuelle Studien zu wichtigen Aspekten der antibiotischen Therapie bei kritisch kranken Kindern beleuchtet und im Hinblick auf klinische
Umsetzbarkeit interpretiert. Folgende Teilaspekte werden besprochen: 1) Zeitpunkt der antibiotischen Therapie und Möglichkeiten eines abwartenden Verhaltens, 2) die Auswahl der Antibiotika in der empirischen Therapie, 3) Deeskalationsstrategien und 4) die Dauer der antibiotischen Therapie. AntibioticStewardship-Programme, unter Einbeziehung von pädiatrischen Infektiologen, klinischen Pharmazeuten und Mikrobiologen, spielen bei den häufig schwierigen klinischen Entscheidungen eine entscheidende Rolle.

Schlüsselwörter

Empirische antibiotische Therapie . Intensivstation · Surviving Sepsis Campaign · Antibiotic Stewardship · Systemic Inflammatory Response Syndrome

\section{Antibiotic treatment in critically ill children-is less more? A narrative review of current studies with reference to the current guidelines of the Surviving Sepsis Campaign}

\section{Abstract}

Antibiotic treatment represents an important and in many cases indispensable treatment to achieve restitutio ad integrum in bacterial infections. Antibiotics can also cure severe infections in immunocompromised patients; however, critically ill children often receive inadequate antibiotic treatment, leading to potentially harmful side effects. The combination of a critically ill child, the fear of missing out on something and the adverse effects of overtreatment complicate rational therapeutic decisions. In this review, we examine and interpret current studies on important aspects of antibiotic treatment in critically ill children with respect to feasibility in the clinical routine. We discuss 1 ) the timing of antibiotic treatment and the possibility to wait and see, 2) the selection of antibiotics for empirical treatment, 3) deescalation strategies, and 4) the duration of antibiotic treatment. Antibiotic stewardship programs, including pediatric infectious disease specialists, clinical pharmacists and microbiologists, play a crucial role in these difficult clinical decisions.

\section{Keywords}

Empirical antibiotic therapy · Intensive care unit · Surviving sepsis campaign · Antibiotic stewardship - Systemic inflammatory response syndrome

\section{Möglichkeiten eines abwartenden Verhaltens}

Eine viel beachtete monozentrische Studie auf einer chirurgischen Erwachsenenintensivstation leistete einen wichtigen Beitrag zur Diskussion über den idealen Zeitpunkt des Therapiebeginns: In einem quasiexperimentellen Ansatz mit Vorher-nachher-Design wurden Daten aus 2 verschiedenen Perioden ausgewertet, in denen jeweils ein unterschiedliches Vorgehen gewählt wurde. Im ersten Zeitraum wurden Patienten auf der Intensivstation mit Verdacht auf eine Infektion sofort und aggressiv antibiotisch behandelt; im zweiten Zeitraum wurden zunächst Kulturen abgenommen und erst 
bei gesicherter Infektion wurde antibiotisch behandelt. Im zweiten Zeitraum war sowohl die Dauer der antibiotischen Therapie (17,7 vs. 12,5 Tage) als auch die adjustierte Mortalität signifikant reduziert (OR 2,5 für Versterben bei aggressiver antibiotischer Therapie (95\%-KI 1,4-4,0)) [15]. Als Grund wurde die häufiger von Beginn an adäquate antimikrobielle Therapie sowie deren kürzere Dauer angesehen. Da diese Studie prospektiv, aber nicht randomisiert kontrolliert durchgeführt wurde und zunächst der aggressive Arm gewählt wurde, muss auch ein Lerneffekt im Erkennen von schweren Infektionen durch das behandelnde Team diskutiert werden. Bei kritisch kranken Patienten mit Verdacht auf eine Sepsis ist ein abwartendes Verhalten nach aktueller Datenlage dennoch kein empfohlenes Vorgehen.

Eine in JAMA Pediatrics publizierte retrospektive Kohortenstudie bei über 10.000 Frühgeborenen $<1500 \mathrm{~g}$ zeigt ebenfalls eine Assoziation zwischen schlechtem Outcome und nichtindizierter antibiotischer Therapie: Antibiotische Therapie ohne Vorliegen einer nekrotisierenden Enterokolitis oder kulturell nachgewiesener Sepsis war mit erhöhter Sterblichkeit verbunden [44]. Möglicherweise führte jedoch die Gabe von Antibiotika ohne bewiesene Infektion nicht per se zu einem schlechteren Outcome, sondern fungierte als Surrogatparameter für mit der Frühgeburtlichkeit assoziierte Komplikationen. Eine effektive Strategie, um die empirische Antibiotikagabe bei Neugeborenen $\mathrm{zu}$ reduzieren und somit unnötige Therapien zu vermeiden, ist die Nutzung eines Onlinerechners, der die Wahrscheinlichkeit für das Vorliegen einer Infektion berechnet und eine Handlungsempfehlung gibt [31]: Eine Metaanalyse über den Effekt des Neonatal Early-Onset Sepsis Calculator (EOS-Calculator) auf die Häufigkeit von Antibiotikagaben zeigte eine Reduktion des relativen Risikos für eine antibiotische Therapie zwischen 3 und $60 \%$ [1]. Der Anteil fälschlich nichtidentifizierter Infektionen betrug bei konventionellem Vorgehen $29 \%$ und bei Nutzung des EOS-Calculators $28 \%$ [1]. Hier wurde zwar kein abwartendes Verhalten untersucht, dennoch zeigen die Ergebnisse, dass es Potenzial zur Reduktion von Antibiotikatherapien auch im Bereich der neonatologischen Intensivmedizin gibt.

Unklar ist, ob bei Verdacht auf eine bakterielle Infektion auch bei kritisch kranken Kindern unter bestimmten Umständen abgewartet werden kann. Der Ansatz wird unterstützt durch retrospektive Studien zu Bakteriämien im Kindesalter, in denen die inadäquate empirische antibiotische Therapie kein direkt nachweisbarer Risikofaktor für schlechtes Outcome war [3, 45, 51]. Diese Strategie - „watchful waiting“ genannt - kann aus klinischer Erfahrung erleichtert werden, wenn rund um die Uhr Kulturen gewonnen und angelegt sowie zeitnah abgelesen werden können, um positive Befunde schnellstmöglich auszugeben. Die Surviving Sepsis Campaign hat 2020 in einem Update zur Therapie der sepsisassoziierten Organdysfunktion und zum septischen Schock die zeitlichen Vorgaben der antibiotischen Therapie präzisiert: Im septischen Schock wird die Gabe innerhalb der ersten Stunde gefordert, bei der sepsisassoziierten Organdysfunktion wurde ein Zeitfenster von $3 \mathrm{~h}$ gewährt, um diagnostische Maßnahmen mit anschließend zielgerichteter Therapie zu ermöglichen.

\section{Auswahl der Antibiotika in der empirischen Therapie}

Die richtige Auswahl der kalkulierten empirischen Therapie einer bakteriellen Infektion wird durch die Zunahme an Infektionen mit multiresistenten Erregern erschwert. Eine Möglichkeit, diesem Problem v. a. bei nosokomialen Infektionen zu begegnen, ist die Wahl einer Kombinationstherapie, d.h. in der Regel ein $\beta$-Lactam mit breitem Spektrum (Penicillin/ $\beta$-Lactamase-InhibitorKombinationen, 3. und 4. Generation Cephalosporine oder Carbapeneme) in Kombination mit einem Aminoglykosid oder mit einem Glykopeptid.

Bei erwachsenen Patienten mit schwerer Sepsis oder septischem Schock wurde beim randomisierten kontrollierten Vergleich einer Monotherapie mit Meropenem mit einer Kombinationstherapie aus Meropenem und Moxifloxacin kein signifikanter Unterschied im primären Zielkriterium Organdysfunktion gesehen. Allerdings hatte diese Studie nicht die Erweiterung des Wirkspektrums, sondern eine Erhöhung der Bakterizidie zum Ziel [6]. Die Hinzunahme von Vancomycin oder Aminoglykosiden zielt hingegen eher auf eine Erweiterung des Wirkspektrums entweder im grampositiven (Vancomycin) oder im gramnegativen (Aminoglykoside) Bereich.

Cephalosporine der 3. Generation in Verbindung mit Gentamicin als kurze Add-on-Therapie bei Sepsis (im Median 2 Tage), verglichen mit einer Cephalosporinmonotherapie, zeigten in einer prospektiven Studie bei Erwachsenen in den Niederlanden ein signifikant erhöhtes adjustiertes Risiko für Nierenversagen, bei nicht signifikant unterschiedlicher Schockdauer und 14-Tage-Mortalität. Die empirische Therapie war in beiden Armen nur in einem sehr geringen Prozentsatz (4\%) inadäquat, sodass die Ergebnisse nicht ohne Weiteres auf Bereiche oder Länder mit höheren Inzidenzen an multiresistenten Erregern übertragbar sind [28].

In der pädiatrischen Literatur zeigen sich inkonsistente Ergebnisse. In einer retrospektiven Studie zur Inzidenz einer akuten Nierenschädigung bei einer Kombinationstherapie aus einem Pseudomonas-wirksamem $\beta$-LactamAntibiotikum und Vancomycin hatte die Kombination mit Piperacillin/ Tazobactam ein signifikant erhöhtes Risiko [10]. Die Effektivität (Reduktion von Mortalität oder Dauer des Aufenthalts im Krankenhaus) wurde hier nicht untersucht. $\mathrm{Zu}$ einem ähnlichen Ergebnis kommt eine weitere retrospektive Studie aus den USA. Die Kombinationstherapie aus $\beta$-Lactam-Antibiotikum und Aminoglykosid hatte eine OR von 2,2 (95\%KI 2,1-2,2) für akute Nephrotoxizität bei gleicher 30-Tage-Mortalität im Vergleich zur alleinigen Therapie mit $\beta$-Lactamen [43]. Die gleiche Arbeitsgruppe konnte auch für schwer kranke Kinder („pediatric risk of mortality score III“ $\geq 15$ ) oder Patienten in Neutropenie keinen Vorteil in Bezug auf die 10-Tages-Mortalität erkennen. Ein signifikanter Überlebensvorteil wurde jedoch bei Patienten mit 
Hier steht eine Anzeige.

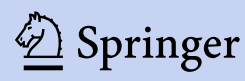


multiresistenter gramnegativer Bakteriämie gesehen [40].

Die Herausforderung hierbei ist, schon bei der empirischen Therapie Patienten mit einem erhöhten Risiko für eine Infektion mit multiresistenten Erregern $\mathrm{zu}$ identifizieren, ohne den übrigen Patienten Antibiotika mit einem ungünstigen Nebenwirkungsprofil $\mathrm{zu}$ verabreichen. Eine theoretische Möglichkeit hierfür sind Kolonisationsscreenings, die teilweise bereits in der Praxis implementiert worden sind. Die Vorhersagbarkeit von gramnegativen Bakteriämien durch Screening auf Besiedelung im Gastrointestinaltrakt schwankt je nach Prävalenz multiresistenter Erreger erheblich. Bisher wurde noch kein Überlebensvorteil durch Screeningmaßnahmen nachgewiesen. Eine 2018 erschienene Metaanalyse zum prädiktiven Wert von Screeninguntersuchungen in der Neonatologie zeigte einen Zusammenhang zwischen Besiedelung mit gramnegativen Bakterien und Blutstrominfektionen. Allerdings war die Qualität der untersuchten Daten nicht ausreichend, und die Ergebnisse waren nicht eindeutig genug, um ein generelles Screening zu empfehlen [12]. Multiresistente Erreger werden meist nur aus Gründen der Krankenhaushygiene nachgewiesen. Eine allgemeine Empfehlung, diese Befunde in die kalkulierte Therapie einzubeziehen, kann aktuell nicht gegeben werden und bleibt eine Einzelfallentscheidung.

Die bereits zitierte Surviving-SepsisCampaign-Leitlinie gibt bei sepsisassoziierter Organdysfunktion oder septischem Schock eine schwache Empfehlung gegen eine Kombinationstherapie bei immunkompetenten Kindern ohne erhöhtes Risiko für multiresistente Erreger und eine schwache Empfehlung für eine Kombinationstherapie bei immunsupprimierten Kindern.

\section{Resistenzgerechte Anpassung und frühzeitiges Beenden von antibiotischer Therapie}

Antibiotika werden bei Verdacht auf eine bakterielle Infektion verordnet. Im klinischen Verlauf bestätigt sich optimalerweise durch kulturellen Nachweis dieser
Verdacht, und die Therapie wird fortgeführt. Oft bestätigt sich der Verdacht nicht, doch die antibiotische Therapie wird trotzdem nicht beendet. Unsicherheit oder Angst der behandelnden Ärzte spielt bei kritisch kranken Kindern hierfür eine wesentliche Rolle.

Bei Kindern mit Fieber in der Neutropenie zeigte eine randomisierte Studie, dass bei viraler Infektion der Atemwege frühzeitig ohne Risiko die empirisch begonnene antibiotische Therapie beendet werden kann [33]. In einer niederländischen prospektiven Multizenterstudie wurden Kinder mit Fieber in der Neutropenie in 3 Gruppen mit jeweils hohem, mittlerem und niedrigem Risiko für eine bakterielle Infektion stratifiziert [26]. Patienten mit klinischen Zeichen einer bakteriellen Infektion oder beeinträchtigten Vitalparametern im Sinne einer Sepsis wurden in die Hochrisikogruppe eingeordnet und antibiotisch behandelt. Anhand des IL-8-Werts wurden die übrigen Patienten in solche mit niedrigem bzw. mittlerem Risiko für eine bakterielle Infektion unterteilt. Patienten in der Niedrigrisikogruppe wurden stationär beobachtet und nach $12 \mathrm{~h}$ Fieberfreiheit entlassen. Patienten in der mittleren Risikogruppe erhielten Antibiotika und wurden nach $72 \mathrm{~h}$ reevaluiert. Nach der Reevaluation wurde bei $41 \%$ der Kinder die antibiotische Therapie beendet, ohne dass es zu einem Rückfall kam. In der Niedrigrisikogruppe hatten 6 Patienten (13\%) eine Infektion mit Koagulase-negativen Staphylokokken, die letztendlich doch antibiotisch behandelt wurde. Die Autoren schlussfolgern, dass es in der mittleren Risikogruppe sicher ist, Antibiotika zu beenden, wenn die Sicherheitsmerkmale erfüllt sind. Die Definition der Niedrigrisikogruppe, die keine Therapie erfordert, stellt hingegen eine Herausforderung dar.

Eine wichtige Strategie ist die resistenzgerechte Anpassung (Deeskalation) nach Erhalt des Resistogramms. Neben der Verschmälerung des Wirkspektrums, die überwiegend auf die Vermeidung von Resistenzen abzielt, wird so auch die Medikamententoxizität für den einzelnen Patienten reduziert. Außerdem wird auf diese Weise die wirksamste vorhandene Therapie gewählt. Den Effekt einer
Deeskalation der antibiotischen Therapie untersuchte eine spanische Gruppe prospektiv an Erwachsenen mit schwerer Sepsis oder septischem Schock. Bei empirisch richtig behandelten Patienten war die Deeskalation nach Erhalt der Blutkultur mit Resistogramm ein protektiver Faktor im Hinblick auf die 90-Tage-Mortalität. Anders ausgedrückt: Von den initial „richtig“ behandelten Patienten starben mehr Patienten, wenn die richtige, aber mit zu breitem Spektrum gewählte Therapie nicht angepasst wurde [13]. Die Surviving Sepsis Campaign empfiehlt die tägliche Evaluation der antibiotischen Therapie im Hinblick auf eine mögliche Deeskalation.

\section{Dauer der Antibiotikagabe}

In der Einleitung wurde bereits darauf eingegangen, dass Antibiotika häufig zu lange verabreicht werden. In diesem $\mathrm{Ab}$ schnitt sollen aktuelle vorwiegend randomisierte Studien besprochen werden, die sich mit der Dauer der antibiotischen Therapie bei intraabdominellen Infektionen befasst haben, weil diese Infektionen besonders gut untersucht sind. Anschließend wird die Wertigkeit des Biomarkers PCT für die Dauer der antibiotischen Therapie diskutiert.

Neben einer antibiotischen Therapie ist bei intraabdominellen Infektionen (Perforation, Abszess u. a.) die operative Sanierung für den Krankheitsverlauf wesentlich. Die Dauer der postoperativen antibiotischen Therapie war Gegenstand einer randomisierten Studie bei Erwachsenen [34]: Verglichen wurde eine Dauer von 2 Tagen nach Resolution von Fieber, Leukozytose und Ileus (Maximum 10 Tage) mit einer fixen Dauer von $4 \pm 1$ Tag. Bezüglich des kombinierten Endpunktes Tod, rekurrierende intraabdominelle Infektion und Infektion des Operationsgebietes gab es keinen signifikanten Unterschied. Der Unterschied in der medianen Dauer der antibiotischen Therapie betrug 4 vs. 8 Tage. $\mathrm{Zu}$ einem ähnlichen Ergebnis kommt eine Beobachtungsstudie [47] an über 1000 Erwachsenen mit komplizierter Appendizitis. Untersucht wurde der Einfluss der Dauer der Antibiotikatherapie nach Appendektomie (3 vs. 5 Tage). Auch 


\section{Infobox 1 Mögliche Quali- tätsindikatoren für pädiatrische Antibiotic-Stewardship-Program- me}

1. Blutkulturen abnehmen: Wichtig ist es, die für die jeweiligen Altersstufen empfohlenen Blutvolumina einzuhalten, um die Wahrscheinlichkeit einer Erregerisolierung zu erhöhen. Diese müssen nicht unbedingt peripher gestochen sein, sondern können auch aus zentralen Kathetern abgenommen werden (Empfehlung GPOH/DGPI)

2. Kulturen aus primär sterilen Körperstellen gewinnen, die als Fokus infrage kommen (z. B. Liquor, Urin, Pleurasekret, Aszites u.a.)

3. Verordnung von Antibiotika nach Leitlinien, die anhand von nationalen Leitlinien erstellt und an die lokale Resistenzlage angepasst wurden

4. Wechsel von empirischer auf gezielte Therapie nach Erregernachweis

5. Anpassen der Dosis an die Nierenfunktion

6. Wechsel von i.v.-Anwendung auf orale Applikation, wenn möglich

7. Erstellen eines Antibiotikaplans: Dieser beinhaltet die Dokumentation der Indikation/Diagnose (z. B. Pyelonephritis, nicht CRP-Erhöhung), Name des Antibiotikums mit Dosierung und Intervall, Weg der Applikation sowie die geplante Dauer der Therapie

8. Antibiotisches Drugmonitoring für Aminoglykoside und für Vancomycin

9. Beenden einer antibiotischen Therapie, wenn sich die bakterielle Infektion nicht bestätigt. In der Regel sollte nach 48-72 h die klinische und mikrobiologische Situation reevaluiert und dokumentiert werden

in dieser Studie konnte kein signifikanter Unterschied auf die Entwicklung tiefer oder oberflächlicher abdomineller Infektionen gesehen werden.

Doch wie ist die Datenlage bei Blutstrominfektionen? In einer retrospektiven „Matched-pair“-Untersuchung wurde die Dauer der antibiotischen Therapie bei Kindern mit unkomplizierter gramnegativer Bakteriämie untersucht. Es gab keine signifikanten Unterschiede zwischen einer 10- und 14-tägigen Therapie, allerdings einen Trend $\mathrm{zu}$ gehäuften Candidämien unter dem längeren Therapieregime [30]. Auch in einer prospektiven randomisierten kontrollierten Studie bei Erwachsenen mit unkomplizierter gramnegativer Bakte-
Weiterführende Informationen zum Thema

Surviving Sepsis Campaign: www.sccm.org AWMF-Leitlinie Sepsis jenseits der Neonatalperiode: https://www.awmf.org/ leitlinien/detail/I//024-025.html

AWMF-Leitlinie Infektionen bei onkologischer Grunderkrankung: https://www.awmf.org/ leitlinien/detail/II/048-014.html

AWMF Leitlinie Bakterielle Infektionen bei

Neugeborenen:

https://www.awmf.org/leitlinien/detail/Il/ 024-008.html

Neonatal Early-Onset Sepsis Calculator: https://neonatalsepsiscalculator. kaiserpermanente.org

riämie waren ein CRP-gesteuertes bzw. 7-Tage-Regime einem 14-Tage-Regime nicht unterlegen. Die Therapiedauer in der CRP-gesteuerten Gruppe betrug im Median 7 Tage [8].

Doch nicht immer ist die standardisierte Therapiedauer ausreichend, z. B. bei ausgeprägter Immunsuppression. Ein Ansatz für eine individualisierte Therapie ist die serielle Bestimmung des PCT unter antibiotischer Therapie. Die Wertigkeit des PCT hinsichtlich der Diagnose einer bakteriellen Infektion ist bereits gut untersucht [27]. Im Folgenden sollen Studien vorgestellt werden, die sich mit der PCT-gesteuerten Dauer der antibiotischen Therapie befassen.

In einer Metaanalyse [49] konnte PCT als ein sensitiver $(0,77$ [95\%KI $0,72-0,81])$ und spezifischer $(0,79$ [95\%-KI 0,74-0,84]) Marker zur Differenzierung zwischen einer Sepsis und einer nichtbakteriellen systemischen Inflammationsreaktion bestätigt werden. Eine weitere Metaanalyse von 26 randomisierten Studien zur PCT-gesteuerten Therapie respiratorischer Infektionen zeigte, dass die Exposition gegenüber Antibiotika von 8 auf 5,7 Tage gesenkt werden konnte [38]. Insbesondere zeigten sich auch eine signifikante Reduktion der Sterblichkeit mit einer OR von $0,83(95 \%$-KI $0,70-0,99)$ sowie eine Reduktion von Nebenwirkungen durch Antibiotika. In den aktuellen AWMFLeitlinien für die ambulant erworbene unkomplizierte Pneumonie im Kindesalter wird bereits eine Behandlungsdauer von 5 Tagen empfohlen. Bemerkenswert ist jedoch, dass in der beschriebenen Metaanalyse auch Studien mit Patienten auf der Intensivstation oder mit nosokomialer Pneumonie eingeschlossen wurden, sodass auch bei nosokomialer Pneumonie eine kurze antibiotische Behandlungsdauer möglich sein könnte (Ausnahmen sind z.B. Infektionen mit Staphylococcus aureus oder Pseudomonas aeruginosa).

Bei Erwachsenen mit schwerer Sepsis oder septischem Schock konnte in einer randomisierten Studie durch Bestimmung des PCT keine Reduktion der Mortalität erwirkt werden, allerdings eine signifikant verkürzte Exposition gegenüber antimikrobiellen Substanzen um $4,5 \%$ [5]. Gleichermaßen verkürzte sich in einer multizentrischen randomisierten kontrollierten Studie die Dauer der Antibiotikatherapie bei Neugeborenen mit Verdacht auf „Early-onset"-Sepsis signifikant von 65 auf $55 \mathrm{~h}$ unter PCTSteuerung. Das frühzeitigere Absetzen führte nicht zu vermehrten Reinfektionen. Eingeschlossen waren allerdings nur Neugeborene mit einer unwahrscheinlichen oder möglichen Infektion, sodass diese Studie primär darauf ausgelegt war, die klinische Entscheidung durch den Einsatz von Biomarkern zu unterstützen. Nicht untersucht wurde die Fragestellung, ob PCT die Dauer einer antibiotischen Therapie bei nachgewiesener oder hinreichend vermuteter Infektion effektiv verkürzen kann [42]. Zudem wird im klinischen Alltag in vielen neonatologischen Abteilungen schon jetzt ein Biomarker-gestützter Algorithmus genutzt, der innerhalb der ersten $48 \mathrm{~h}$ zu einer Beendigung der Therapie führt (persönliche Kommunikation). Häufig wird bei Verdacht auf Early-onset-Sepsis postnatal IL-6 oder IL- 8 bestimmt, und im Verlauf (nach 24h) CRP. Die aktuelle AWMF-Leitlinie Bakterielle Infektionen bei Neugeborenen bescheinigt dieser Kombination eine sehr hohe Sensitivität $(97 \%)$ und Spezifität (94\%) [52].

Die aktuelle Literatur ist dahingehend konsistent, dass mithilfe von PCT der Verbrauch von Antibiotika gesenkt werden kann. So führt (bei negativem Kulturergebnis) ein Abfall bzw. ein fehlender Anstieg jeweils zum Absetzen einer anti- 
biotischen Therapie. In einem klinischen Setting, in dem es eher zu einer Übertherapie kommt (schwer kranke Kinder, Intensivstationen), wird dieser Effekt möglicherweise kleiner, wenn viele Patienten ohne Infektion trotz negativem PCT antibiotisch behandelt werden.

Die aktuelle Surviving-Sepsis-Campaign-Leitlinie empfiehlt, die Dauer der antimikrobiellen Therapie festzulegen, in Abhängigkeit vom Ort der Infektion, dem auslösenden Erreger, dem klinischen Verlauf und der Möglichkeit einer Fokussanierung. Zur Anwendung von PCT hinsichtlich Therapiebeginn oder -dauer gibt es keine Empfehlung.

\section{Annäherung an eine adäquate Antibiotikatherapie durch fachliche Expertise und Antibiotic-Stewardship- Programme}

Die Sorge, eine bakterielle Infektion bei kritisch kranken Kindern nicht oder nicht adäquat zu behandeln, führt, wie beschrieben, zu einer Übertherapie - mit ebenfalls unerwünschten Wirkungen. Multiresistente Erreger, invasive Infektionen oder Begleiterkrankungen wie Niereninsuffizienz erschweren die kalkulierte und manchmal auch die gezielte Therapie. Um in diesem Spannungsfeld zu einer optimierten Versorgung zu kommen, haben sich im klinischen Alltag ABS-Programme bewährt. Ein gelebtes ABS-Programm (personell ausgestattet mit pädiatrischem Infektiologen, Mikrobiologen und klinischem Pharmazeuten) kann die Qualität der antibiotischen Therapie verbessern. In einer prospektiven Interventionsstudie in der Neonatologie konnte ein ABS-Programm den Antibiotikaverbrauch (gemessen in Tagen mit Antibiotikatherapie/1000 Patiententagen) signifikant um $27 \%$ senken [7]. Ein systematisches Review über ABSProgramme in der Pädiatrie konnte zeigen, dass diese den Verbrauch von Antibiotika in Kinderkliniken senken [2]. Allerdings wurde im Bereich der pädiatrischen Intensivmedizin in keiner Studie ein Effekt nachgewiesen [2]. Als ursächlich diskutieren die Autoren, dass die Anwesenheit von Fremdmaterial und die schwere Grunderkrankung die An- wendung von Antibiotika mit schmalem Spektrum erschweren. Dennoch ist auch im Bereich der pädiatrischen Intensivmedizin davon auszugehen, dass ABSProgramme die Qualität der Antibiotikaverordnung erhöhen.

Diese Qualität kann anhand von Indikatoren gemessen werden, wie in einer aktuellen Publikation dargestellt [37]. Beispielhaft sind hier einige Indikatoren genannt, die für die Erwachsenenmedizin vorgeschlagen werden und von den Autoren für die Pädiatrie angepasst wurden (- Infobox 1).

Die flächendeckende Implementierung von ABS-Programmen sowie die regelmäßige Überprüfung ausgewählter Qualitätsindikatoren im stationären Alltag könnten dazu beitragen, Antibiotika rationaler anzuwenden, ohne dadurch Patienten zu gefährden.

\section{Fazit für die Praxis}

- Die im Titel gestellte Frage lässt sich derzeit nicht endgültig beantworten.

- Kinder mit febriler Neutropenie, septischem Schock und Organversagen profitieren von einer frühzeitigen und aggressiven antibiotischen Therapie.

- Bei Patienten ohne Organdysfunktion erscheint ein abwartendes Verhalten in manchen Fällen vertretbar.

- Die Entscheidung, ob eine empirische Therapie um ein Aminoglykosid oder Glykopeptid erweitert wird, bleibt eine Einzelfallentscheidung unter Abwägung des potenziellen Nutzens gegenüber dem bekannten Nebenwirkungsprofil einzelner Substanzen. Eine wesentliche Herausforderung in diesem Zusammenhang stellt die Identifizierung von Patienten mit invasiven Infektionen durch multiresistente Erreger dar.

- Unabhängig von der gewählten empirischen Therapie ist eine frühzeitige Reevaluation mit Anpassung oder Beendigung der Antibiotkatherapie immer indiziert.

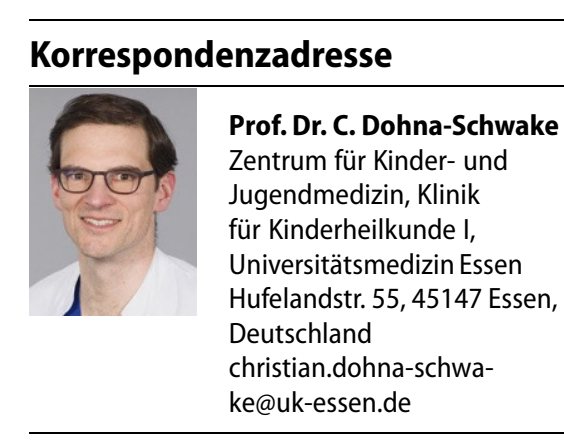

Funding. Open Access funding enabled and organized by Projekt DEAL.

\section{Einhaltung ethischer Richtlinien}

Interessenkonflikt. N. Bruns und C. Dohna-Schwake geben an, dass kein Interessenkonflikt besteht.

Für diesen Beitrag wurden von den Autoren keine Studien an Menschen oder Tieren durchgeführt. Für die aufgeführten Studien gelten die jeweils dort angegebenen ethischen Richtlinien.

Open Access. Dieser Artikel wird unter der Creative Commons Namensnennung 4.0 International Lizenz veröffentlicht, welche die Nutzung, Vervielfältigung, Bearbeitung, Verbreitung und Wiedergabe in jeglichem Medium und Format erlaubt, sofern Sie den/die ursprünglichen Autor(en) und die Quelle ordnungsgemäß nennen, einen Link zur Creative Commons Lizenz beifügen und angeben, ob Änderungen vorgenommen wurden.

Die in diesem Artikel enthaltenen Bilder und sonstiges Drittmaterial unterliegen ebenfalls der genannten Creative Commons Lizenz, sofern sich aus der Abbildungslegende nichts anderes ergibt. Sofern das betreffende Material nicht unter der genannten Creative Commons Lizenz steht und die betreffende Handlung nicht nach gesetzlichen Vorschriften erlaubt ist, ist für die oben aufgeführten Weiterverwendungen des Materials die Einwilligung des jeweiligen Rechteinhabers einzuholen.

Weitere Details zur Lizenz entnehmen Sie bitte der Lizenzinformation auf http://creativecommons.org/ licenses/by/4.0/deed.de.

\section{Literatur}

1. Achten NB, Klingenberg C, Benitz WE et al (2019) Association of use of the neonatal early-onset sepsis calculator with reduction in antibiotic therapy and safety: a systematic review and metaanalysis. JAMA Pediatr 173:1032. https://doi.org/ 10.1001/jamapediatrics.2019.2825

2. Araujo da Silva AR, Albernaz de Almeida Dias DC, Marques AF et al (2018) Role of antimicrobial stewardship programmes in children: a systematic review. J Hosp Infect 99:117-123. https://doi.org/ 10.1016/j.jhin.2017.08.003

3. Averbuch D, Avaky C, Harit M et al (2017) Nonfermentative Gram-negative rods bacteremia in children with cancer: a 14-year single-center experience. Infection 45:327-334. https://doi.org/ 10.1007/s15010-017-0988-1 
Hier steht eine Anzeige.

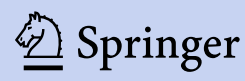


4. Blinova E, Lau E, Bitnun A et al (2013) Point prevalence survey of antimicrobial utilization in the cardiac and pediatric critical care unit. Pediatr Crit Care Med 14:e280-e288. https://doi.org/10. 1097/PCC.0b013e31828a846d

5. Bloos F, Trips E, Nierhaus A et al (2016) Effect of sodium selenite administration and procalcitoninguided therapy on mortality in patients with severe sepsis or septic shock: a randomized clinical trial.JAMA Intern Med 176:1266-1276. https://doi. org/10.1001/jamainternmed.2016.2514

6. Brunkhorst FM, Oppert M, Marx G et al (2012) Effect of empirical treatment with moxifloxacin and meropenem vs meropenem on sepsis-related organ dysfunction in patients with severe sepsis: a randomized trial. JAMA 307:2390-2399. https:// doi.org/10.1001/jama.2012.5833

7. Cantey JB, Wozniak PS, Pruszynski JE, Sánchez PJ (2016) Reducing unnecessary antibiotic use in the neonatal intensive care unit(SCOUT): a prospective interrupted time-series study. Lancet Infect Dis 16:1178-1184. https://doi.org/10.1016/S14733099(16)30205-5

8. von Dach E, Albrich WC, Brunel A-S et al (2020) Effect of C-reactive protein-guided antibiotic treatment duration, 7-day treatment, or 14-day treatment on 30-day clinical failure rate in patients with uncomplicated gram-negative Bacteremia: a randomized clinical trial. JAMA 323:2160-2169. https://doi.org/10.1001/jama.2020.6348

9. Di Pentima MC, Chan S, Hossain J (2011) Benefits of a pediatric antimicrobial stewardship program at a children's hospital. Pediatrics 128:1062-1070. https://doi.org/10.1542/peds.2010-3589

10. Downes KJ, Cowden C, Laskin BL et al (2017) Association of acute kidney injury with concomitant vancomycin and Piperacillin/Tazobactam treatment among hospitalized children. JAMA Pediatr 171:e173219. https://doi.org/10.1001/ jamapediatrics.2017.3219

11. Folgori L, Livadiotti S, Carletti M et al (2014) Epidemiology and clinical outcomes of multidrugresistant, gram-negative bloodstream infections in a European tertiary pediatric hospital during a 12-month period. Pediatr Infect Dis J 33:929-932. https://doi.org/10.1097/INF.0000000000000339

12. Folgori L, Tersigni C, Hsia Y et al (2018) The relationship between Gram-negative colonization and bloodstream infections in neonates: a systematic review and meta-analysis. Clin Microbiol Infect 24:251-257. https://doi.org/10.1016/j.cmi.2017. 08.008

13. Garnacho-Montero J, Gutiérrez-Pizarraya A, Escoresca-Ortega A et al (2014) De-escalation of empirical therapy is associated with lower mortality in patients with severe sepsis and septic shock. Intensive Care Med 40:32-40. https://doi. org/10.1007/s00134-013-3077-7

14. Goldstein B, Giroir B, Randolph A, International Consensus Conference on Pediatric Sepsis (2005) International pediatric sepsis consensus conference: definitions for sepsis and organ dysfunction in pediatrics. Pediatr Crit Care Med 6:2-8. https:// doi.org/10.1097/01.PCC.0000149131.72248.E6

15. Hranjec T, Rosenberger LH, Swenson B et al (2012) Aggressive versus conservative initiation of antimicrobial treatment in critically ill surgical patients with suspected intensive-care-unitacquired infection: a quasi-experimental, before and after observational cohort study. Lancet Infect Dis 12:774-780. https://doi.org/10.1016/S14733099(12)70151-2

16. Hufnagel M, Versporten A, Bielicki J et al (2019) High rates of prescribing antimicrobials for prophylaxis in children and neonates: results from the antibiotic resistance and prescribing in European children point prevalence survey. J Pediatric Infect Dis Soc 8:143-151. https://doi. org/10.1093/jpids/piy019

17. Irwin AD, Drew RJ, Marshall P et al (2015) Etiology of childhood bacteremia and timely antibiotics administration in the emergency department. Pediatrics 135:635-642. https://doi.org/10.1542/ peds.2014-2061

18. Ivády B, Kenesei É, Tóth-Heyn P et al (2016) Factors influencing antimicrobial resistance and outcome of Gram-negative bloodstream infections in children. Infection 44:309-321. https://doi.org/10. 1007/s15010-015-0857-8

19. Johnson MT, Reichley R, Hoppe-Bauer J et al (2011) Impact of previous antibiotic therapy on outcome of Gram-negative severe sepsis. Crit Care Med 39:1859-1865. https://doi.org/10.1097/CCM.0b013e31821b85 f4

20. Ko BS, Choi S-H, Kang GH et al (2020) Time to antibiotics and the outcome of patients with septic shock: a propensity score analysis. Am J Med 133:485-491.e4. https://doi.org/10.1016/j. amjmed.2019.09.012

21. Kreitmeyr K, von Both U, Pecar A et al (2017) Pediatric antibiotic stewardship: successful interventions to reduce broad-spectrum antibiotic use on general pediatric wards. Infection 45:493-504 https://doi.org/10.1007/s15010-017-1009-0

22. Kumar A, Roberts D, Wood KE et al (2006) Duration of hypotension before initiation of effective antimicrobial therapy is the critical determinant of survival in human septic shock. Crit Care Med 34:1589-1596. https://doi.org/10.1097/01.CCM. 0000217961.75225.E9

23. Levy M, Kolodziejczyk AA, Thaiss CA, Elinav E (2017) Dysbiosis and the immune system. Nat Rev Immunol 17:219-232. https://doi.org/10.1038/ nri.2017.7

24. Livorsi D, Comer A, Matthias MS et al (2015) Factors influencing antibiotic-prescribing decisions among inpatient physicians: a qualitative investigation. Infect Control Hosp Epidemiol 36:1065-1072. https://doi.org/10.1017/ice.2015. 136

25. Metjian TA, Prasad PA, Kogon A et al (2008) Evaluation of an antimicrobial stewardship program at a pediatric teaching hospital. Pediatr Infect Dis J 27:106-111. https://doi.org/10.1097/ INF.0b013e318158603a

26. Miedema KGE, Tissing WJE, Abbink FCH et al (2016) Risk-adapted approach for fever and neutropenia in paediatric cancer patients-a national multicentre study. Eur J Cancer 53:16-24 https://doi.org/10.1016/j.ejca.2015.10.065

27. Niehues T (2017) C-reaktives Protein und andere immunologische Biomarker. Monatsschr Kinderheilkd 165:560-571. https://doi.org/10.1007/ s00112-017-0314-0

28. Ong DSY, Frencken JF, Klein Klouwenberg PMC et al (2017) Short-course adjunctive gentamicin as empirical therapy in patients with severe sepsis and septic shock: a prospective observational cohort study. Clin Infect Dis 64:1731-1736. https:// doi.org/10.1093/cid/cix186

29. Park DA, Lee SM, Peck KR et al (2013) Impact of methicillin-resistance on mortality in children and neonates with staphylococcus aureus bacteremia: a meta-analysis. Infect Chemother 45:202-210. https://doi.org/10.3947/ic.2013.45.2.202

30. Park SH, Milstone AM, Diener-West M et al (2014) Short versus prolonged courses of antibiotic therapy for children with uncomplicated Gram- negative bacteraemia. J Antimicrob Chemother 69:779-785. https://doi.org/10.1093/jac/dkt424

31. Puopolo KM, Draper D, Wi S et al (2011) Estimating the probability of neonatal early-onset infection on the basis of maternal risk factors. Pediatrics 128:e1155-e1163. https://doi.org/10.1542/peds. 2010-3464

32. Salstrom JL, Coughlin RL, Pool K et al (2015) Pediatric patients who receive antibiotics for fever and neutropenia in less than $60 \mathrm{~min}$ have decreased intensive care needs. Pediatr Blood Cancer 62:807-815. https://doi.org/10.1002/pbc. 25435

33. Santolaya ME, Alvarez AM, Acuña M et al (2017) Efficacy and safety of withholding antimicrobial treatment in children with cancer, fever and neutropenia, with a demonstrated viral respiratory infection: a randomized clinical trial. Clin Microbiol Infect 23:173-178. https://doi.org/10.1016/j.cmi. 2016.11.001

34. Sawyer RG, Claridge JA, Nathens AB etal (2015) Trial of short-course antimicrobial therapy for intraabdominal infection. N Engl J Med 372:1996-2005. https://doi.org/10.1056/NEJMoa1411162

35. Schlapbach LJ, Straney L, Alexander J et al (2013) Antibiotic consumption and resistance: data from Europe and Germany. Int J Med Microbiol 303:388-395. https://doi.org/10.1016/j. ijmm.2013.04.004

36. Schlapbach LJ, Straney L, Alexander J et al (2015) Mortality related to invasive infections, sepsis, and septic shock in critically ill children in Australia and NewZealand,2002-13:a multicentre retrospective cohort study. Lancet Infect Dis 15:46-54. https:// doi.org/10.1016/S1473-3099(14)71003-5

37. Schlapbach LJ, Straney L, Alexander J et al (2015) Quality indicators to measure appropriate antibiotic use in hospitalized adults. Clin Infect Dis 60:281-291. https://doi.org/10.1093/cid/ciu747

38. Schuetz P, Wirz Y, Sager R et al (2018) Effect of procalcitonin-guided antibiotic treatment on mortality in acute respiratory infections: a patient level meta-analysis. Lancet Infect Dis 18:95-107. https://doi.org/10.1016/S1473-3099(17)30592-3

39. Shanahan F, Sheehan D (2016) Microbial contributions to chronic inflammation and metabolic disease. Curr Opin Clin Nutr Metab Care 19:257-262. https://doi.org/10.1097/MCO. 0000000000000282

40. Sick AC, Tschudin-Sutter S, Turnbull AE et al (2014) Empiric combination therapy for gram-negative bacteremia. Pediatrics 133:e1148-e1155. https:// doi.org/10.1542/peds.2013-3363

41. Singer M, Deutschman CS, Seymour CWetal(2016) The third international consensus definitions for sepsis and septic shock (sepsis-3). JAMA 315:801-810

42. Stocker M, van Herk W, El Helou S et al (2017) Procalcitonin-guided decision making for duration of antibiotic therapy in neonates with suspected early-onset sepsis: a multicentre, randomised controlled trial (NeoPIns). Lancet 390:871-881. https://doi.org/10.1016/S0140-6736(17)31444-7

43. Tamma PD, Turnbull AE, Harris AD et al (2013) Less is more: combination antibiotic therapy for the treatment of gram-negative bacteremia in pediatric patients. JAMA Pediatr 167:903-910. https://doi.org/10.1001/jamapediatrics.2013.196

44. Ting JY, Synnes A, Roberts A et al (2016) Association between antibiotic use and neonatal mortality and morbidities in very low-birth-weight infants without culture-proven sepsis or necrotizing enterocolitis. JAMA Pediatr 170:1181-1187. https:// doi.org/10.1001/jamapediatrics.2016.2132 
45. Tsai M-H, Chu S-M, Hsu J-F et al (2014) Risk factors and outcomes for multidrug-resistant Gram-negative bacteremia in the NICU. Pediatrics 133:e322-e329. https://doi.org/10.1542/peds. 2013-1248

46. Turta O, Rautava S (2016) Antibiotics, obesity and the link to microbes - what are we doing to our children? BMC Med 14:57. https://doi.org/10. 1186/s12916-016-0605-7

47. van Rossem CC, Schreinemacher MHF, Treskes $\mathrm{K}$ et al (2014) Duration of antibiotic treatment after appendicectomy for acute complicated appendicitis. Br J Surg 101:715-719. https://doi org/10.1002/bjs.9481

48. Versporten A, Bielicki J, Drapier N et al (2016) The Worldwide Antibiotic Resistance and Prescribing in European Children (ARPEC) point prevalence survey: developing hospital-quality indicators of antibiotic prescribing for children. J Antimicrob Chemother 71:1106-1117. https://doi.org/10. 1093/jac/dkv418

49. Wacker C, Prkno A, Brunkhorst FM, Schlattmann P (2013) Procalcitonin as a diagnostic marker for sepsis: a systematic review and meta-analysis. Lancet Infect Dis 13:426-435. https://doi.org/10. 1016/S1473-3099(12)70323-7

50. Weiss SL, Fitzgerald JC, Balamuth F et al (2014) Delayed antimicrobial therapy increases mortality and organ dysfunction duration in pediatric sepsis. Crit Care Med 42:2409-2417. https://doi.org/10. 1097/CCM.0000000000000509

51. Weiss SL, Peters MJ, Alhazzani W et al (2020) Surviving sepsis campaign international guidelines for the management of septic shock and sepsisassociated organ dysfunction in children. Pediatr Crit Care Med 21:e52-e106. https://doi.org/10. 1097/PCC.0000000000002198

52. Zemlin M, Berger A, Franz AR et al (2019) AWMFLeitlinie Bakterielle Infektionen bei Neugeborenen (https://www.awmf.org/leitlinien/detail/II/024008.html)

Fachnachrichten

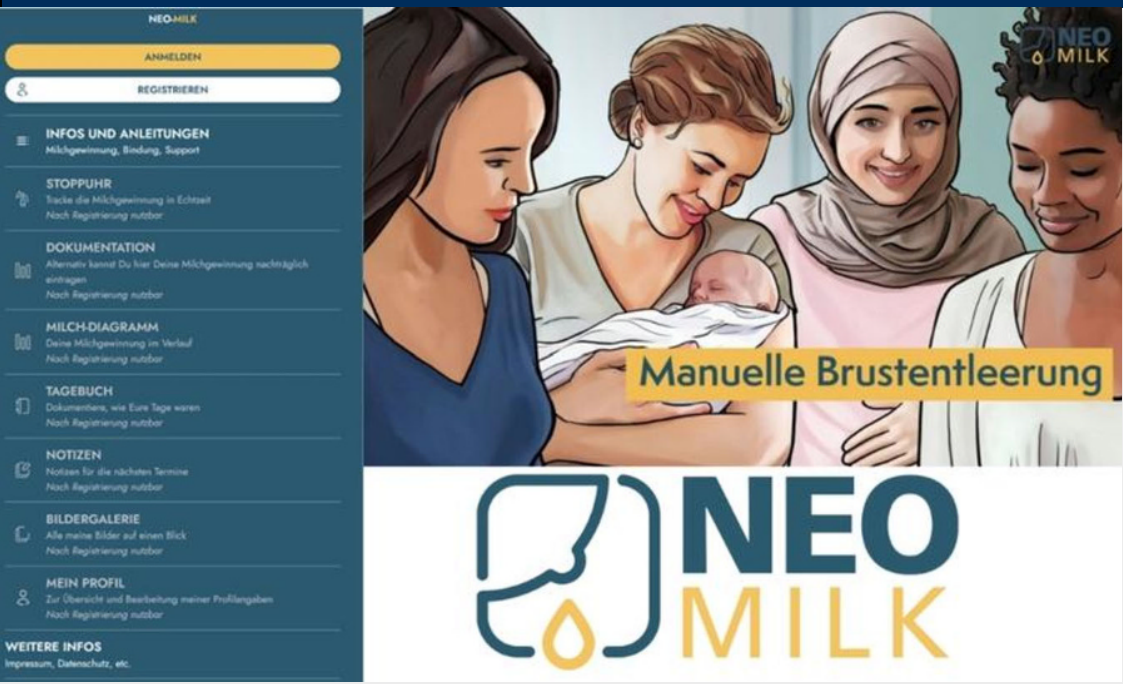

\section{Neo-MILK: Strukturelle Stillförderung und Aufbau von Humanmilchbanken}

Das durch den Gemeinsamen Bundesausschuss (G-Ba) geförderte Projekt zeigt nach der Hälfte der Förderdauer bereits erste Erfolge

Neo-MILK ist ein vom G-Ba gefördertes wissenschaftliches Projekt mit dem Ziel, mehr Frühgeborene mit Muttermilch zu ernähren. Im Vordergrund steht die Optimierung der Stillförderung in deutschen Perinatalzentren. Steht keine oder temporär keine Milch der Mutter zur Verfügung, sollen die Frühgeborenen humane Spenderinnenmilch aus Humanmilchbanken erhalten.

Die Implementierung des evidenzbasierten Neo-MILK Still- und Laktationsförderungskonzeptes wie auch der Aufbau von Humanmilchbanken erfolgt in 15 neonatologischen Intensivstationen; dort wird auch die Evaluation im Rahmen eines Cluster-randomisierten Stepped-Wedge-Designs durchgeführt. Schon nach 2 Jahren Projekt- und Entwicklungszeit (Projektstart 1.1.2021) wurden wichtige Meilensteine erreicht. Eine mit Neonatolog*innen, Stillberater*innen, Psycholog*innen aus 4 Universitäten und Universitätskliniken sowie betroffenen Müttern entwickelte App unterstützt die Mütter bei der Initiierung der Laktation und dem kontinuierlichen Abpumpen. Neben Informationen rund um die Milchgewinnung enthält sie Videos, um die Mütter anzuleiten. Die Kurzfilme, die auch außerhalb der App zugänglich sind, decken folgende Themen ab:

- Manuelle Entleerung der Brust

- Abpumpen mit einer Milchpumpe

- Brustmassage und Steigerung der Milchmenge

- Hygiene und Muttermilch und

- Lösungen und Wege: Herausforderungen beim Stillen.
Die Neo-MILK App kann die Mütter an flexibel festgelegte Abpumpzeiten erinnern. Die abgepumpte Milchmenge kann dokumentiert und der Verlauf grafisch sichtbar gemacht werden.

Die App und die Filme stehen bereits jetzt allen Interessierten kostenfrei zur Verfügung. Flyer zur Neo-MILK App und zu den Filmen können bei uns angefordert werden (Kontakt: neomilk@uk-koeln.de).

\section{Wissenschaftliche Leitung Neo-MILK}

Dr. Till Dresbach (Uniklinik Bonn) und

PD Dr. Nadine Scholten (Uniklinik Köln)

\section{Weitere Informationen}

Unter www.neo-milk.de oder auf Instagram: neo_milk.

Quelle: Neo-MILK, Nadine Scholten 